\title{
DEBATES
}

\section{El cambio climático desde el capital social de mujeres indigena Mapuche}

\author{
Climate change from the social capital of Mapuche indigenous \\ women
}

\section{Carolina Fuentes Lizama}

\section{Resumen}

En la Región de la Araucanía, Chile, las mujeres indígenas Mapuche se han visto seriamente afectadas por eventos extremos del cambio climático. El objetivo del estudio consiste en explorar factores y alternativas de resiliencia que emergen desde sus procesos de empoderamiento para responder ante los efectos del cambio climático, tomando en consideración su rol como productoras y reproductoras de su cultura tradicional. Los resultados indican que las mujeres Mapuche articulan estrategias que les permitan sobrevivir de la mejor forma posible a fenómenos como la sequía o los incendios forestales con la consecuente búsqueda de alternativas de mitigación tales como transformar sus actividades económicas tradicionales en mecanismos operacionales de adaptación al cambio climático. Estos procesos revelan potenciales relevantes para la resiliencia, que debiesen ser considerados por acciones institucionales para la reducción de riesgos, tales como inversiones en infraestructuras críticas.

\section{Palabras claves}

Cambio Climático; Mujeres Indígenas; Cultura Mapuche; Capital Social.

\begin{abstract}
In the Region of Araucanía, Chile, indigenous Mapuche women have been seriously affected by extreme events of climate change. The objective of the study is to explore resilience factors and alternatives that emerge from their empowerment processes to respond to the effects of climate change, considering their role as producers and reproducers of their traditional culture. The results indicate that Mapuche women articulate strategies that allow them to survive in the best possible way to phenomena such as drought or forest fires with the consequent search for mitigation alternatives such as transforming their traditional economic activities into operational mechanisms of adaptation to climate change. These processes reveal relevant potentials for resilience, which should be considered by institutional actions to reduce risks, such as investments in critical infrastructure.
\end{abstract}

\section{Keywords}

Climate Change; Indigenous Women; Mapuche Culture; Social Capital. 


\section{Introducción ${ }^{1}$}

El cambio climático y los riesgos de desastres naturales son fenómenos que generan el desafío de la adaptación para las distintas sociedades y a diferentes escalas (IPCC, 2012). Esto porque los efectos del cambio climático y de los desastres naturales se combinan con decisiones sociales y políticas que predisponen a las sociedades a ser afectadas adversamente por los estímulos del clima. La variabilidad del clima y los eventos extremos afectan especialmente a los países en vías de desarrollo, cuyos sistemas económicos y sociales son más dependientes de los servicios ecosistémicos y presentan mayores tasas de vulnerabilidad. Por tanto, la adaptación al cambio climático consiste también en decisiones sociales y políticas que contribuyan a la reducción de esa vulnerabilidad (VALDIVIESO, 2016).

En Chile, se observan avances en los campos de las ciencias y la tecnología que han permitido identificar los diferentes escenarios posibles ante los efectos del cambio climático para la prevención de desastres naturales. Sin embargo, un parte importante de la población Mapuche de la región de La Araucanía se enfrentan a condiciones de vida que no cumplen con los estándares mínimos, enfrentan pobreza extrema y no poseen una vivienda adecuada que les permita enfrentar los efectos del cambio climático. Lo anterior refleja asimetrías en las relaciones entre la sociedad chilena, el Estado chileno y el Pueblo Mapuche. Este es el principal problema que imposibilita una adaptación resiliente, inclusiva para el mayor bienestar social.

La población indígena Mapuche constituye la más numerosa de las identidades étnicas de Chile. Gran parte de su población vive en zonas rurales que alguna vez fueron de gran riqueza ecológica, pero que hoy están sometidos a estrés debido al impacto de la industria forestal en la zona. En este sentido, los procesos de empoderamiento femenino de las mujeres indígenas del territorio de la Araucanía, nos permite no solo conceptualizar los efectos que tiene el cambio climático en el "mundo Mapuche", sino también los efectos de decisiones y acciones humanas que explican desafíos en sus condiciones de vida.

Si bien el contacto entre españoles primero, y chilenos después, con el mundo indígena generó ciertos cambios en la cultura ancestral, la base de su constitución y de su cosmovisión como Mapuche no varió en esencia y continúa, hasta el día de hoy,

\footnotetext{
${ }^{1}$ La autora agradece las observaciones y recomendaciones de los árbitros anónimos, más las del editor de este número. Asimismo, agradece el apoyo financiero que hace posible actividades de investigación y diseminación científica relacionadas, relevantes para elaborar este estudio: proyecto Fondecyt Conicyt n. 1181282, proyecto UNAP-VRIIP-0169-16.
} 
sustentándose en su propia lógica indígena. En este sentido, es posible observar hoy en día un proceso de revitalización infra pueblo Mapuche a pesar de los embates de la globalización y que se observan en la celebración de ceremonias a lo largo del territorio indígena conocido como "Walmapu"

El pueblo Mapuche se ha configurado desde sus inicios a través de la transmisión oral de sus saberes a las distintas generaciones basándose en la concepción dual de su mundo de la vida. En este sentido, abordar un análisis en torno al rol que juegan las mujeres como mitigadoras de impactos al cambio climático, nos plantea observar el rol que han tenido las mujeres Mapuche históricamente en la lucha reivindicadora por sus derechos y que debe considerar una visión crítica desde las propias mujeres ${ }^{3}$. En este sentido, relacionando conceptos que abordaremos más adelante, partimos considerando que esas dinámicas y procesos de revitalización cultural, y de empoderamiento femenino, manifiestan una forma de capital social.

En este estudio nos proguntamos por el potencial de lo anterior para procesos de adaptación resiliente de las mujeres Mapuche, en el entendido que la resiliencia significa la capacidad de un sistema para responder a estímulos que perturban su funcionamiento y desarrollarse con estabilidad hacia el futuro. Nos preguntamos si en ello se manifiestan activos de capital social, y finalmente nos preguntamos sobre si las políticas de desarrollo territorial debiesen integrar y promover ese potencial para la co-producción de soluciones que permitan una mejor adaptación, por ejemplo mediante inversiones que promuevan infraestructuras críticas y acciones de protección medioambiental informadas por ese saber local.

La hipótesis transversal que se plantea en esta investigación es que las mujeres Mapuche son capaces de enfrentar de mejor forma el cambio climático, ser resilientes, y adaptarse, debido a los procesos de empoderamiento femenino facilitados por la concentración de capital social, principalmente del tipo bonding, con un fuerte sentido de "nosotros" (LIN, 2008).

La investigación que ha dado origen a este estudio es de tipo interpretativa, sigue un diseño triangulado que pone especial énfasis en la perspectiva de los sujetos que participan de la investigación - Mujeres Mapuche. En este sentido, el trabajo está

\footnotetext{
${ }^{2}$ Nombre dado al territorio ancestral habitado históricamente por los Mapuche.

${ }^{3}$ Por ejemplo, en su momento la poligamia no era vista como algo problemático y aunque las culturas son dinámicas, no debemos adjetivar con nuestras propias conceptualizaciones occidentales en los sentires y saberes de esas mujeres.
} 
ubicado dentro de la línea argumentativa que indica que la participación y colaboración permite obtener mejores resultados ya que la visión de los distintos sujetos y actores locales otorgan sentido al trabajo realizado (PEREDA et al., 2008).

El estudio se realizó gracias a la colaboración de una agrupación de mujeres Mapuche, compuesta por cerca de 80 socias, quienes representan los desafíos y sueños de las mujeres del territorio. El trabajo de campo se realizó durante distintos periodos desde el año 2015 y las técnicas para la obtención de datos fue observación participante en sus actividades cotidianas, entrevistas en profundidad y la realización de dos talleres de grupos focales. Con posterioridad, el trabajo de campo ha sido complementado mediante un permanente contacto con las mujeres y sus comunidades. En las entrevistas se abordó temas tales como la percepción del cambio climático, así como las consecuencias para su forma de vida en las zonas rurales de La Araucanía.

\section{Cambio climático, ecologia y adaptación}

Abordamos el marco teórico de este estudio desde la perspectiva ecológica del desarrollo, por ser una aproximación cercana a las cosmovisiones y representaciones que declaran los pueblos indígenas. Esta perspectiva nos permite comprender de mejor forma al sujeto en estudio, las mujeres Mapuche, y sus adaptaciones en un contexto de constante cambio.

Desde la discusión epistemológica se ha buscado dar respuesta a la pregunta por el lugar que ocupa el ser humano dentro de los (eco) sistemas siendo la ecología la disciplina que nos ha aportado respuestas al respecto (PENGUE, 2012). La ecología, como ciencia que estudia las relaciones entre organismos vivientes y su medio, observa con especial cuidado las relaciones que se generan entre diferentes especies para comprender la forma en que son capaces de organizarse para subsistir (GUDYNAS, 2004).

En este sentido se entiende que el ser humano, a diferencia de los animales, es capaz de modificar su medioambiente a través de la cultura y de esa manera adaptarse a los diferentes medios que les parezcan más convenientes, ya que, se observa a la naturaleza desde una matriz de creencias y conocimientos que permean la concepción de lo real (GEERTZ, 1994).

Como plantean Castro y Zusman (2009), la naturaleza aparece aquí como un instrumento material útil para el progreso de la humanidad y como una herramienta funcional para la construcción del poder económico que viene a justificar la categorización que se realiza de ella. No obstante, se comienza a cuestionar la 
transformación y el dominio de la naturaleza por el ser humano y comienza una revaloración de la "naturaleza salvaje", observando lo natural de manera dicotómica, pero ya no como un bien material dispuesto para la utilización del hombre (ALIATA y SILVESTRI, 1994; COATES, 1998).

Bajo el contexto del cambio climático, el agotamiento de los recursos y el aumento de la contaminación ambiental, el planteamiento sobre el dominio y domesticación de la naturaleza comienza a ser fuertemente criticado en la década de 1960, centrando su atención en el abuso de productos químicos y la expansión demográfica depredadora de recursos naturales. Surge también la inquietud por el desigual acceso a las tecnologías por parte de los llamados países periféricos (CASTRO y ZUSMAN, 2009).

En este período se continúa tratando la cuestión de la naturaleza y la cultura de manera dicotómica, pero con una mirada crítica sobre las acciones del ser humano. En este contexto re-aparecen las ideas de la filosofía de la naturaleza para respaldar de alguna forma la idea que la explotación social y ambiental generadas por el capitalismo son la causa de la pérdida de los recursos naturales (O'CONNOR, 2001). También aparecen más tarde, y con mucha fuerza, los lineamientos de la ecología política latinoamericana, que tiene como principal argumento la influencia del trauma de la conquista en las relaciones de poder como mediadoras en la relación naturaleza/sociedad, siendo la naturaleza necesaria para la acumulación de capital (KEUCHEYAN, 2014; LEFF, 2003).

La perspectiva ecológica es crítica frente a todos los desarrollos sintetizados, y parte por buscar un desarrollo que reconozca el sentido que dan los propios sujetos a sus contextos y experiencias en los complejos sistemas socioambientales. Desde la cosmovisión indígena, la proximidad que se da entre los componentes culturales y la naturaleza difieren enormemente de la forma de relación que el mundo occidental ha tenido con el medio ambiente a lo largo de su historia. Por esta razón, nosotros abordamos este estudio con la premisa que es necesario entender distinciones o elementos de esa cosmovisión para, en etapas posteriores, estar en condiciones de promover acciones de desarrollo, reducción de riesgos de desastres, y adaptación al cambio climático que sean consistentes con la cultura Mapuche. 


\section{Capital social como factor explicativo de adaptación al cambio climático}

Dentro del desarrollo de las comunidades, la organización y las relaciones sociales reiteradas han jugado un rol preponderante para asegurar su éxito en diferentes proyectos comunitarios o para resolver sus problemas durante momentos críticos, como, por ejemplo, cuando ocurren catástrofes naturales (VALDIVIESO y DAVIDOVICS, 2016). Para los científicos sociales esta forma de vínculos constituye una forma de capital que permite a las comunidades desarrollar sus actividades colectivas y salir adelante de manera exitosa (OSTROM, 1990; GREEN y HAINES, 2008). Sin embargo, existe una amplia brecha en el acceso a oportunidades entre quienes carecen de recursos y quienes los concentran, por lo que un número no menor de población queda al margen y no puede generar estrategias asertivas para paliar los efectos del cambio climático (COLLIER, 1998).

Ante esta realidad, el reforzamiento de la identidad y el empoderamiento han emergido como medios que permiten facilitar el acceso a recursos que de otra forma las comunidades, sobre todo las mujeres indígenas, no podrían tener, debido a su condición de marginalidad y exclusión (AHMAD y HALL, 2012).

Por tanto, consideraremos la discusión sobre el capital social y las redes que poseen los grupos es relevante, porque estos activos pueden ser determinantes para entender de qué manera las comunidades hoy en día, y específicamente las comunidades que habitan las mujeres Mapuche de La Araucanía, son capaces de adaptarse a los efectos del cambio climático, ser resilientes y una fuente de conocimiento para aportar al desarrollo territorial.

Como plantean Gary Green y Anna Haines, las relaciones sociales pueden ser consideradas como una forma de capital debido a que es necesario invertir tiempo y energía para disfrutar de esos recursos, obtener retornos, además de que tienen la propiedad de mejorar la calidad de vida de quien hace uso de ellos, ser acumulables y productivos (GREEN y HAINES, 2008). La Organización para la Cooperación y el Desarrollo Económico (OECD), en tanto, define capital social como "el conjunto de redes, normas, valores y entendimientos compartidos que facilitan la cooperación en o entre grupos" (OECD, 2007, p. 102). Para Narayan, el capital social se define como las relaciones sociales y las normas que se encuentran integradas en las estructuras sociales y que les permiten a las personas generar acciones para alcanzar sus propósitos (NARAYAN, 1999).

De acuerdo a Ram y Prasad (2008), los diferentes estudios que se han realizado sobre acciones colectivas, vinculadas principalmente al manejo de los 
recursos naturales, utiliza el concepto del capital social desde la visión propuesta por Putnam (1993), debido a que el autor establece una relación directa entre la acumulación de capital social y el nivel organizacional de un grupo, argumentando que los colectivos pueden resolver sus problemas gracias a este capital facilitador. Putnam además utiliza el concepto del capital social para analizar el desempeńo de diferentes instituciones como por ejemplo los gobiernos regionales (RAM y PRASAD, 2008).

Desde esta perspectiva, podemos establecer tres unidades de análisis que han sido ilustradas por diferentes autores, las cuales son: capital social bonding, bridging y linking (NARAYAN y CASSIDY, 1999; AHMAD y HALL, 2012; GREEN y HAINES, 2012; ANDERSSON y VALDIVIESO, 2017).

Los términos bonding y bridging fueron introducidos en la discusión por Putnam (1993) y Gittel y Videl (1998), quienes demostraron que las diferentes variables del capital social permiten caracterizar las dimensiones sociales de una comunidad (AHMAD y HALL, 2012). Putnam, al tratar estas dos dimensiones de capital social, define el bridging como vínculos y conexiones entre diferentes tipos de personas (PUTNAM, 2000). Por otro lado, el capital social bonding se refiere a vínculos que posee personas o grupos de personas que se conocen y relacionan entre sí de un modo más directo y frecuente, por ejemplo, los vínculos que se establecen en una familia, parientes o grupos étnicos, permitiéndoles reafirmar la identidad de grupo (PUTNAM, 2000). Para el autor, el capital social bonding permite asegurar la reciprocidad y solidaridad. Por otro lado, el capital social bridging permite generar vínculos importantes con agentes fuera del propio grupo, accediendo a información y estableciendo redes que pueden garantizar desarrollo y bienestar (KNUDSEN et al., 2008). Putnam señala que ambos son muy importantes, sobre todo a nivel local, ya que pueden contribuir al crecimiento económico de un territorio propiciando un mejoramiento en la calidad de vida de las personas (PUTNAM, 2000).

Según Uphoff (1996), esto se debe a que una de las principales características del capital social bonding es el compartir cultura y tradición dentro de un colectivo como un conjunto de normas o tradiciones que permiten sobrevivir, ya que los problemas que les afectan son de interés de todas las personas de determinado grupo y sus proyecciones futuras son similares (UPHOFF, 1996 apud RAM, 2008).

Por otro lado, Wolff y Wahab determinaron que es posible encontrar, dentro de las organizaciones indígenas, mayor predominancia a la sustentabilidad que las comunidades no indígenas, ya que sus miembros son capaces de establecer relaciones 
de confianza duraderas y a largo plazo, tanto en la comunidad como también hacia sus líderes, quienes son elegidos de acuerdo con su sabiduría y su experiencia (WOLFF y WAHAB, 1996 apud RAM, 2008).

En síntesis, cuando hablamos de capital social, en contexto de las mujeres Mapuche de La Araucanía, nos referimos al capital social bonding como el conjunto de normas, roles y responsabilidades, basadas en la confianza, y que permiten la realización de acciones colectivas por parte de los grupos que poseen ese capital.

La atención en esta forma de capital compartida por miembros de un mismo grupo, identidad y/o colectividad, constituye sin duda el enfoque apropiado para comprender la manera en que, desde los campos de La Araucanía las mujeres Mapuche levantan estrategias de adaptación a los efectos del cambio climático. Por otra parte, el enfoque se fortalece cuando nos apoyamos para abordar la problemática desde la epistemología y la ecología, ya que nos permite identificar factores y/o situaciones que pueden ser explicadas desde una mirada más integral que relaciona el capital bonding con la forma de relación naturaleza-cultura que se da sobre todo en los pueblos indígenas.

En los siguientes apartados sintetizaremos hallazgos de nuestro estudio.

\section{E1 clima y la visión que tienen las mujeres Mapuche al respecto}

La mujer Mapuche tiene una visión respecto de los efectos de los diversos extremos y desastres naturales debido a su cosmovisión indígena.

Para el Mapuche el agua no es sólo dadora de vida, sino es vida en sí, tiene esencia o espíritu, el Ngenko, por tanto es un Newen o energía, forma parte fundamental de nuestra cosmovisión. Siendo así, no puede existir sola, tiene que ser en simbiosis con otros elementos y/o Newen, constituyendo el Itrofillmongen o Biodiversidad (DOMINGO RAIN, 2007, p. 1).

El clima de la región de La Araucanía, en el Sur de Chile, favorece el desarrollo de una gran variedad de vegetación y especies únicas. El clima se define como de tipo templado lluvioso con una oscilación térmica que rodea los $8^{\circ} \mathrm{C}$. Se caracteriza además por inviernos lluviosos con precipitaciones que bordean los 1.250 $\mathrm{mm}$ concentrada en los meses de junio, julio y agosto, seguidos de veranos cálidos menos lluviosos (40 mm). 
Debido a que el cambio climático es un fenómeno visible a escala global es difícil que las mujeres puedan percibirlo de manera precisa, por lo tanto, en las entrevistas a las se les solicitó identificar algunos cambios y/o variaciones de las que ellas pudiesen dar cuenta. Cabe destacar que, dentro de la lengua vernácula del pueblo Mapuche, el mapudungun, no existe el cambio climático ya que al ser un término contemporáneo no se encuentra incorporado en el vocabulario. Por tanto, se buscaron algunas correspondencias que permitieran mostrar los cambios que ocurren en el clima y los efectos que ello tiene en su población.

Desde las mujeres Mapuche, el cambio climático se relaciona directamente con la falta de agua en las comunidades, de lo cual responsabilizan a la industria forestal tal como muestra el testimonio.

\footnotetext{
"En mi comunidad no tenemos agua potable, toda la vida hemos tenido que arreglárnosla con el agua de pozo. Pero este verano que pasó hizo tanto calor que los pozos bajaron mucho y ya no se puede sacar mucha agua de ahí...la municipalidad nos trae mil litros a la semana y con eso nos la tenemos que arreglar para cocinar, usar el bańo, lavar la ropa, todo. El agua de pozo la usamos solo para regar la huerta, porque si no ahí si que estaríamos mal, pero usted ve allá, al otro lado los eucaliptus de la forestal, tremendos de grande, se llevaron toda el agua, y nos dejan a nosotros sin nada" (Entrevistada n. 1, 12 feb. 2015).
}

Para las mujeres Mapuche, el cambio climático también se refiere a temperatura y las lluvias o la falta de ellas. En este sentido, la percepción de ellas es que existe mucha lluvia en el invierno que no es aprovechada de ninguna forma, ya que no cuentan con los medios para capturarla, seguida por períodos prolongados de sequía, entre los meses de diciembre a abril, que son devastadores para ciertas cosechas.

De acuerdo con el informe de la Dirección Meteorológica de Chile, el déficit hídrico en la región de La Araucanía llega a cerca del 57\% siendo los años 2013 y 2015 considerados como los años más secos en más de 50 años. Por lo tanto, es posible señalar una correspondencia entre lo que las mujeres Mapuche perciben y los datos climatológicos disponibles.

Uno de los puntos más relevantes es entender que los cambios del clima afectan y tienen graves consecuencias en la seguridad alimentaria de las comunidades Mapuche, y del mundo en general. Desde la Organización de las Naciones Unidas para la Alimentación y la Agricultura se define a la seguridad alimentaria como el 
acceso físico y/o económico a todos los alimentos nutritivos, inocuos y suficientes para satisfacer las necesidades dietéticas de una población para asegurar su vida activa y saludable (FAO, 2007).

En este sentido, los cambios en el clima impactan directamente a todos los componentes del sistema alimentario de las familias Mapuche. La base de su alimentación es una combinación de cereales y legumbres, junto a carne de animales domésticos como las gallinas, los cerdos, el cordero y los vacunos. Para las mujeres, la falta de agua a causa de la sequía, les afecta en todo el desarrollo de sus actividades diarias, ya que dependen de lo que siembran y de los animales que crían. Por lo tanto, en la medida en que las cosechas se vean afectadas, ellas sentirán los efectos del clima.

"Ahora ya no sale a cuenta plantar trigo, uno ya no sabe como viene el
tiempo, entonces pasa que un verano llueve un poco y el trigo sale
lechoso y ya no sirve. Aparte que el precio que pagan es muy poco, no
vale la pena, por eso estamos plantando avena y pasto para forraje pa'l
invierno, pero sino hay que arrendar el terreno no más, a los que tienen
más recursos para hacerlo andar y le ponen riego tecnificado y les sale de
todo" (Entrevistada n. 3, 22 de feb. 2015).

En cuanto a la percepción por la temperatura, las mujeres indicaron que sienten que los inviernos están cada vez más fríos con mucha helada y los veranos demasiados calurosos. Ellas opinan que la cantidad de incendios forestales que se observan en el verano, indican cuando el tiempo está muy caluroso.

Desde la percepción de las mujeres entrevistadas los efectos de las temperaturas se pueden observar en las transformaciones del paisaje. En palabras de una entrevistada, mujer Mapuche de la comunidad de Lincay:

“[...] cuando era nińa recuerdo que yo me arrancaba de la casa a jugar en un tranque que formaba justo en un riachuelo que hay cerca de la casa. En el tranque había muchas ranas y a mí me encantaba sentarme ahí y las escuchaba cantar en la tarde. De a poco el tranque se fue achicando y un verano muy caluroso que hubo se fue toda el agua. Todas las ranitas se murieron y quedaron secas ahí en la tierra dura. La gente dice que la sequía es cosa de ahora no más y yo pienso que están equivocados porque esto se arrastra desde hace mucho tiempo antes [...]" (Entrevistada n. 5, 20 jun. 2015).

Dentro de los cambios que ellas observan se encuentran, además, la depredación de sus animales domésticos por animales que viven en los montes, 
muerte de gallinas por zorros y pumas, así como también la pérdida progresiva del lawen, plantas para usos medicinales. Ellas señalan que hay muchas especies que ya no es posible encontrar en los alrededores de sus comunidades y que deben ir a otros territorios a buscarlas. Lamentan además que el puma, conocido en el sector como "el león", baje desde el lugar donde habita a alimentarse de las gallinas que poseen. Ellas reconocen que los animales lo hacen porque ya no hay que comer producto que muchos animales se han muerto por falta de agua.

\begin{abstract}
"[...] si usted me pregunta cuales son los cambios o como dice los efectos, es que ha habido pérdidas muy grandes, porque ya la tierra ya no da. O sea imagínese que sale más barato comprar la harina lista que trabajar toda la temporada, porque tampoco no hay seguridad pa' uno ¿y si todo se pierde? Este año el trigo se subió y salió todo lechoso, no sirve y esa tierra ya no sirve para nada hay que esperar otra temporada, porque pa' abajo está todo tan caliente que se quema, y a lo más se puede plantar avena pero eso no más, antes no porque antes uno se turnaba y plantaba trigo y porotos y uno tenía de todo pal invierno [...]" (Entrevistada n. 3, 22 de feb. 2015).
\end{abstract}

De igual forma, para las mujeres Mapuche, los cambios que han ocurrido en el campo a causa del cambio del clima han tenido un impacto a nivel emocional, ya que la falta de agua, a causa de la sequía ha significado que muchas familias deban separarse; los hombres migran a otros territorios en busca de trabajo, mientras que sus hijos viven de lunes a viernes en modalidad internado. Tal como se observa en el siguiente testimonio.

\footnotetext{
“[...] a diferencia de hace 40 años, hoy en día el campo está envejecido, ya no hay nińos ni jóvenes quienes se han marchado a las ciudades a estudiar como internos y solo van a casa los fines de semana. Los hombres se van al norte a trabajar de temporeros y eso ha llevado a que muchos no han vuelto, pues han rehecho su vida lejos. Las mujeres están solas. El campo hoy día tiene rostro de mujer" (ONG CHOL-CHOL, 8 jun. 2015).
}

Lindley sugiere que el tema del desplazamiento producto de la sequía no es responsabilidad absoluta de las variaciones climáticas que afectan al planeta sino más bien obedecen a la poca o nula capacidad de respuesta de los gobiernos locales para hacer frente a los problemas de la población entendidas en términos de asegurar 
alimentación, vivienda y agua. En palabras de la autora, "cuando se permite que una sequía se desarrolle y desemboque en una grave crisis, los esfuerzos humanitarios se ven obstaculizados por la politización de la ayuda y la corrupta economía política que la envuelve" (LINDLEY, 2014, p. 42).

Este punto cobra mucha importancia cuando les consultamos a las mujeres por la forma en que ellas han visto que ha cambiado su comunidad por el cambio climático. Entre las principales respuestas destaca la sensación de miedo a estar sola, sin apoyo en caso que algo ocurra en el campo, por ejemplo, que muera un animal o que ocurra un incendio.

\section{De las estrategias de adaptación}

Para comprender las estrategias de adaptación de las mujeres Mapuche de la Araucanía a los eventos extremos del cambio climático, es necesario señalar, que, de acuerdo con la tradición, al momento de contraer matrimonio ellas deben dejar sus comunidades de origen y se trasladan a vivir a la comunidad de sus esposos. En un primer momento esto es problemático porque la obliga a tejer toda una nueva red de apoyo en el nuevo territorio que habita. Por lo tanto, debe desarrollar estrategias de adaptación con los recursos disponibles en la nueva comunidad donde está.

Las familias en el campo no pueden subsistir sólo en base a los animales de corral o la horticultura, sino que dependen en gran medida de las cosechas anuales. Las épocas de siembra y cosecha le significan a la mujer Mapuche dedicación a diferentes labores en las cuales se mezcla la tradición cultural con la recolección anual. El trigo es el principal sustento para las familias y la tarea de su mantención queda legada a los hombres. Las mujeres se dedican a alimentar a sus esposos y a las personas que ayuden en los procesos de arado, siembra y cosecha.

Además, muchas han recurrido a la elaboración de artesanía textil para complementar sus ingresos con lo que produce el campo. Esto ha llevado a que las mujeres se muevan entre lógicas de tiempo y espacio diferentes (urbano/rural moderno/no moderno) bajo la necesidad de producir y vender para sobrevivir. Mientras en su casa se dedican a cuidar a sus animales y la huerta, en la ciudad tienen que lidiar con los medios de transporte, la comunicación satelital, el uso del dinero, el manejo de tendencias en moda y el marketing.

Las mujeres realizan prácticas colectivas que necesariamente dependen de la confianza y reciprocidad mutuas y que se basan principalmente en la cosmovisión. En este punto cabe destacar el mecanismo que les permite a las mujeres contar con los alimentos necesarios cuando hay sequía. 


\begin{abstract}
"Nosotros aquí tenemos un chiquillo que trabaja en la micro que va para Rulo, entonces yo llamo al super, al lili y le digo a una nińa que trabaja ahí también que necesito no se fideos, carne o cualquier cosa y ella me lo anota y lo lleva al terminal y el Juan me lo echa arriba de la micro y yo salgo al camino a buscar la mercadería. Así nos ha ayudado mucho, cuando salió malo el trigo, tuvimos que hacerlo así, porque no teníamos para hacer el pan" (Entrevistada n. 4, 18 feb. 2015).
\end{abstract}

$\mathrm{Al}$ consultar a las mujeres cómo se hacen frente a estos cambios que vienen dados por la deslocalización de las actividades económicas por el cambio climático podemos destacar que en general se ubican dentro de lo que se entiende como multi estrategia:

a. Se produce movilidad campo-ciudad, principalmente hombres que salen en busca de trabajo, dejando a las mujeres en casa. Como nos seńala una entrevistada "hoy en día hay mucha mujer como jefa de hogar, da igual si tiene o no tiene marido, porque ella es la que lleva el pan a la casa” (Entrevistada n. 8, 24 jul. 2015);

b. Se genera un giro en la actividad económica, pasando de la dedicación casi exclusiva en la agricultura a complementarse con producción de artesanías y venta de animales y hortalizas en el pueblo más cercano.

Para las mujeres de La Araucanía, y suponemos que en otros territorios también, la producción de artesanías se ha convertido en un mecanismo de sobrevivencia que les ha permitido generar ingresos económicos para sostener el hogar en un contexto marcado por la sequía y en que las ganancias por la producción agrícola se hacen cada vez más escasa. Surge el concepto de trabajo en la mujer Mapuche, concepto que en la década de los 1990 estaba delegado exclusivamente para las mujeres que abandonaban el campo y viajaban a la ciudad a trabajar como empleadas domésticas. Por un lado, las mujeres asumen la jefatura del hogar y por otro se enfrentan a esta nueva realidad, que a su vez enfrenta una serie de desafíos debido a los impactos que acarrea la globalización a través de la reorganización espacial de producción de mercado que genera cambios en la forma de competir (MITTELMAN, 1997).

La proliferación de factores adversos en un contexto geofísico complejo, marcado por los eventos extremos y el cambio climático en La Araucanía, ha creado las condiciones para la proliferación de un mercado específico permitiendo que la 
producción de artesanías se constituya como una estrategia interesante a través de la generación de ingresos económicos.

A este respecto les consultamos a las mujeres que colaboraron con esta investigación ¿Cómo enfrentan estos cambios? y ellas nos señalan que:

a. Las productoras de artesanías han buscado aprovechar las ventajas que les trae la globalización para el desarrollo de su actividad, principalmente a través del uso de herramientas comunicacionales propias del siglo XXI, como la internet;

b. La agrupación con pares que realizan la misma actividad como una estrategia que les permita visibilizar su quehacer y además entrar en otros mercados más competitivos, por ejemplo, las ventas al sector retail que demandan mayores volúmenes de producción.

Dentro de los testimonios de artesanas encontramos:

"Soy hija de artesana, aprendí a tejer a los 8 años, mi mamá y mi abuela se encargaron de enseñarme y de traspasarme los conocimientos en telar Mapuche. Mi mamá también fue la que me enseñó a vender mi primer tejido, eso fue a los 12 ańos y de ahí en adelante me dediqué a producirlos y venderlos, ya que eso me significó ganarme mi propio dinero y comprar las cosas que yo necesitaba" (Entrevistada n. 3, 22 feb. 2015).

"Yo aprendí a tejer a través de mi mamá. Ella tejía a telar y yo era muy inquieta entonces también aprendí así mirando. Yo pasaba de chica recogiendo la lana por los alambres que quedaban ahí donde la oveja iba pasando yo llegaba y la guardaba y la hilaba. Al principio mi mami me regalaba los hilos después me dijo que tenía que hacerlos yo. Cuando tenía como 13 ańos me dio un capital, eran como cuatro kilos de lana y lo tuve que lavar hilar y tejer. Hice una frazada y mi papá fue a Temuco y la vendió, el me apoyaba harto me decía hágalo usted solita y yo se lo voy a vender. Nosotros éramos hartos hermanos porque mi papi tenía dos esposas, es que él era cacique le llaman ahora. Después yo me fui a la capital a trabajar como empleada, fui a la misma casa que trabajaba mi hermana, pero no me halle, lloraba todos los días y la patrona era media pesada, y dije ¿Qué estoy haciendo acá si 94 puedo estar allá y ganar igual plata? Y me volví y me puse a trabajar en esto hasta hoy día” (Entrevistada n. 1, 12 feb. 2015).

Relevando estos relatos recordamos lo que nos plantean Tellería y Conde (2004) y Salanova y Cabañés (2010), quienes señalan que es necesario problematizar hoy en día la forma en que los productores de artesanías han aprovechado las ventajas 
de la globalización y de estos procesos de intensificada industrialización en contexto de sequía, utilizando las tecnologías de la información para salir del ámbito de lo local y posicionarse en diferentes escenarios. También nos señalan la importancia de estar atentos a la sobreexposición de estos conocimientos, estos bienes que solían tener un valor exclusivamente de uso y que ahora son apetecidas mercancías por diferentes usuarios, ya que éstos pueden llevar a la desterritorialización del mismo lo cual hace que se pierda el contenido del objeto.

$\mathrm{Al}$ respecto consultamos a una entrevistada y ella comenta que:

\footnotetext{
"Nuestro gran desafío es ¿Cómo compatibilizamos el gusto de las personas (por la artesanía) hoy en día con nuestra cultura ancestral? Para nosotros es muy importante continuar produciendo tejidos, porque llevamos el sustento a los hogares, con nuestro trabajo le compramos zapatos a nuestros hijos, un mes le compramos a uno, al otro mes al otro y así... yo creo que de lo que producimos el $90 \%$ va para el hogar, el otro $10 \%$ lo ocupamos para traslados y poder llegar a vender a otras partes, o sea que al final es el 100\%” (Entrevistada n. 3, 22 feb. 2015).
}

Como bien señala la entrevistada, y como recoge el trabajo La mujer en la agricultura Chilena (2009) elaborado por el Instituto Nacional de Estadísticas, la participación de la mujer en actividades laborales remuneradas en el campo, tales como el área silvoagropecuaria, es escasa debido a la división sexual del trabajo en el cual las mujeres se encargan de las tareas domésticas y el cuidado de los miembros del hogar (INE, 2009) aportando casi todo sus ingresos percibidos por otras actividades económicas en beneficio directo de su hogar, ya sea para comprar alimentos, ropa e incluso para comprar el alimento de los animales. Es en este contexto de marcada necesidad, en el cual la sequía constituye el vector que materializa esta estrategia de producción de artesanías como alternativa para obtener ingresos.

\section{Reflexiones finales y conclusiones}

Los testimonios y evidencias de nuestro estudio sugieren que el capital social bonding explica la resiliencia y es una ventana de oportunidades para progresar en la adaptación, y debiese ser promovido y fortalecido por intervenciones y políticas territoriales que tengan el propósito de contribuir al bienestar

En los campos de la Araucanía las mujeres Mapuche han asumido diversos roles que les han permitido mantener viva la cultura y las tradiciones de generación 
en generación, roles que se han complejizado a raíz de las condiciones de vida extremas ocasionadas por el cambio climático.

Recordemos que desde el trabajo de Robert Putnam entendemos el concepto de capital social bonding como vínculos que posee un grupo de personas que se conocen y relacionan entre sí, los cuales les permite reafirmar su identidad como colectivo (PUTNAM, 2000). Bajo esta mirada, observando, consultando y relacionando informaciones, establecemos y reafirmamos la hipótesis inicial con que partimos esta investigación, afirmando que las mujeres Mapuche de la Araucanía son capaces de generar estrategias de adaptación debido a que poseen una importante concentración de capital social bonding.

Esta forma de capital se manifiesta en las acciones que toman parte de la vida en comunidad, la preservación de la lengua y las tradiciones propias bajo una visión del ser Mapuche, elemento identitario central para entender la resiliencia de las mujeres mapuches.

En el entendido que los seres humanos tenemos una dimensión individual y otras relacional, social y territorial, como condiciones básicas para el desarrollo de las personas, podemos profundizar un poco más en el Ser Mapuche. Cuando hablamos de cultura Mapuche debemos remitirnos al pasado para comprender. Como nos señala Magnus Course en su trabajo titulado Los géneros sobre el pasado en la vida rural Mapuche (2010), no podemos entender esta idea del "ser" como la simple acumulación de vidas individuales, sino como una concatenación de existencias en función de un contexto dado (COURSE, 2010). Mapuche significa "gente de la tierra” y esto, más allá de su concepción literal, hace referencia a una manera específica de vivir, de habitar un territorio y de proyectar sueños y deseos.

En un contexto pre-eminentemente rural, la pregunta por la identidad cultural, por el ser Mapuche, viene determinada en parte por los modos de asentamiento agrícola que de alguna manera han reemplazado los modos de subsistencia que se basaban en la combinación de horticultura, caza y pesca. Por esta razón, ser Mapuche hoy en día implica diferenciarse de la población Chilena (Winka = nuevo inka) en una suerte de entendimiento cuasi marxista de identidad de clases, en la cual la sociedad se encuentra dividida por clases que luchan por el control, en este caso, de los recursos naturales (COURSE, 2010).

Las posibilidades de ser se configuran a partir de diferentes ámbitos ya sea territorial, cultural, religioso, político, económico. Debemos considerar que no existe una única definición correcta de lo que es ser Mapuche. Pero este estudio nos ha 
permitido revisar algunas concepciones que emergen desde la propia voz de las mujeres.

Dentro de las respuestas que emergieron desde ellas surgen con fuerza los conceptos de: tierra, lengua y costumbres como los elementos identitarios más importantes. En la cosmovisión Mapuche, el ser humano y la tierra conforman parte de un todo.

El capital social bonding aparece entonces como un factor explicativo de la resiliencia de las mujeres Mapuche, en un contexto de cambios importantes debido a los eventos extremos y la escasez. Este tipo de capital está representado por las dinámicas de participación y de vida comunitaria que se generan al interior de los territorios en los que habitan las entrevistadas, quienes comparten como común denominador: los problemas derivados de la sequía. Dentro de los hallazgos más importantes destacamos:

a. La relación que las mujeres tienen con los miembros de su comunidad viene determinada en gran parte por el parentesco. Ellas dejaron sus territorios de nacimiento y se trasladaron a vivir con sus esposos, generando alianzas intercomunidades, lo cual permite la participación de muchas personas durante tiempos de cosechas, junto con abaratar costos en caso de necesitar comprar o arrendar alguna maquinaria. Además, el hecho de que su comunidad tenga la "calidad" indígena, la hace un elemento diferenciador respecto del no mapuche.

Como hemos mencionado anteriormente, la relación del ser Mapuche viene dada en gran parte en base a la diferenciación entre quienes son Mapuche y quienes no lo son. Por otro lado, el idioma como elemento constitutivo de la cultura tiende a presentarse difuso en gran parte de las entrevistadas, debido principalmente a que es un componente que se ha perdido por el desuso. Sin embargo, ellas relevan su valor y la importancia que tiene que los niños la aprendan, ya que es la única forma que tienen los Mapuche para poder dotar de significado su territorio, en el sentido más estricto;

b. La gran mayoría de las comunidades en las que habitan las mujeres que colaboraron con este trabajo se reúnen periódicamente. Como mínimo realizan reuniones una vez al mes, las cuales tienen el carácter de informativas sobre proyectos y otras materias que para sus ellas son importantes, como consultas indígenas. Esto permite que las personas estrechen lazos y se sientan más identificados con los problemas del otro, generándose grupos de personas que comparten ideales, sentires y sueños comunes. 
Esto viene determinado principalmente por el fuerte sentido de confianza y reciprocidad entre vecinos; es muy usual escuchar a una mujer decir "hoy día hice tortillas así que voy a llevarle estas a la María porque tiene hijos chicos y parece que su marido no trajo na' moneas" (Entrevistada n. 1, 12 feb. 2015).

Este punto es muy importante para comprender como funciona la comunidad ya que a la hora de resolver problemas relacionados con el cambio climático las comunidades son capaces de trabajar, colaborar y desarrollar relaciones de reciprocidad. En palabras de una entrevistada del sector rural Lliuco:

[...] esa vez que llovió como una semana sin parar parece que acá llueve más que en Temuco porque se llegó a cortar el camino y al final entre todos pusimos madera para hacer un puentecito improvisado, y después le pedimos por medio del presidente de la comunidad que se comunicara con la Muni para pedir una retro pero nunca llegó así que entre todos pusimos la plata y arrendamos una, nos costó como 30 mil, pero había que hacerlo [...] (Entrevistada n. 4, 18 feb. 2015).

Al ser consultadas si en sus comunidades están preparadas y/o cuentan con planes de acción elaborados de manera conjunta para hacer frente a los efectos del clima, las personas entrevistadas coincidieron en afirmar que ese tema es algo que nunca se habla en las reuniones, ya que por lo general a la gente le interesa saber sobre proyectos para el campo. Aún así, muchas concuerdan que es una buena idea elaborar una planificación conjunta.

Desde nuestra perspectiva, la posibilidad de que los grupos elaboren estrategias de desarrollo y/o estrategias preventivas a largo plazo permiten fortalecer el tejido social de los mismos, ya que es posible identificar situaciones y soluciones para problemas específicos y de esa manera resolverlos en menor tiempo y con una tasa de éxito más alta que aquellos grupos que no se encuentran cohesionados. Esta es una estrategia de adaptación efectiva, según testimonia la experiencia de las mujeres Mapuche;

c. Situándonos desde la hipótesis de nuestra investigación que afirma que las mujeres Mapuche de La Araucanía son capaces de generar alternativas y estrategias de adaptación al cambio climático debido a que poseen un capital bonding, podemos decir que las redes de apoyo con las que cuentan estas mujeres son muy fuertes, ya que esos bonds se encuentran sobre la base de la familia nuclear pero también de la familia extendida a través de lazos intra-comunitarios, junto con el fuerte sentido de identidad Mapuche. El capital social bonding, se encuentra fortalecido debido al 
sentido que tiene ser Mapuche y viene a jugar un rol fundamental a la hora de entender por qué las mujeres de estos grupos son capaces de permanecer en el campo, aun cuando la sequía y los incendios forestales amenazan constantemente.

Finalmente, como implicaciones de todo lo anterior, nuestras evidencias indican que una adecuada estrategia de adaptación territorial al cambio climático debe integrar a la mujer Mapuche con su capital bonding como agente relevante para co-producir bienes públicos esenciales para el bienestar. En este sentido, las instituciones que tienen responsabilidad en el desarrollo territorial debiesen promover esa forma de capital para colaborar en decisiones y acciones que fortalezan la resiliencia. Por ejemplo, iniciativas y proyectos de infraestructuras, tales como sistemas de riego para la agricultura, que integren esas prácticas y formas de articulación social que quedan en evidencia en este estudio. En este sentido, una tarea de nuestra futura agenda de investigación consiste en entender las lógicas institucionales para identificar ventanas de oportunidades que permitan avanzar en esa decisión, e informar a los tomadores de decisión.

Carolina Fuentes Lizama é Mestre em Ciências Sociais. Pesquisadora e Professora da Universidad Arturo Prat. E-mail cafuentes@unap.cl.

\section{Referências}

AHMAD, Mahyudin; HALL, Stephen. Can trust explain social capital effect on property rights growth? In: International conference on economics and business research. 2012. Disponível em: <http://www.sciencedirect.com/science/article/pii/S2212567113002189>. Acesso em: 22 mar. 2018.

ALIATA, Fernando; SILVESTRI, Gabriela. El paisaje en el arte y las ciencias humanas. Centro Editor de América Latina: Buenos Aires. 1994.

ANDERSSON, Krister; VALDIVIESO, Patricio. Why local governments matter: adapting to a changing climate in Chile. Harvard Rev. Lat. Am., v. 37, n. 6, p. 1916-33, 2017.

CASTRO, Hortencia; ZUSMAN, Perla. Naturaleza y cultura: ¿dualismo o hibridación? Una exploración para los estudios sobre riesgo y paisaje desde la Geografía. Investigaciones Geográficas, n. 70, p. 135-153, 2009.

COATES, Jennifer. Language and gender: a reader. Oxford. 1998. 
COLLIER, Paul. Social capital and poverty en Social Capital initiative. Working Paper. n. 4. 1998. Disponível em: <http://siteresources.worldbank.org/INTSOCIALCAPITAL/Resources/SocialCapitalInitiative-Working-Paper-Series/SCI-WPS-04.pdf>. Acesso em: 22 mar. 2018.

COURSE, Magnus. Los géneros sobre el pasado en la vida Mapuche Rural. Revista Chilena de Antropología. n. 21. Universidad de Chile. 2010. Disponível em: $<$ http://www.revistadeantropologia.uchile.cl/index.php/RCA/article/viewFile/14108/14412>. Acesso em: 5 maio 2018.

FAO. Food and Agriculture Organization. Cambio climático y seguridad alimentaria: Un documento marco. 2007. Disponível em: <http://bvssan.incap.int/local/cambio-climatico/CAMBIOCLIMATICO-INSAN-MARCOFAO.pdf>. Acesso em 23 maio 2018.

GEERTZ, Clifford. La interpretación de las culturas. Ed. Gedisa. 1994.

GITTEL, Ross; VIDEL, Avis. Social capital and networks in community development: framing the LISC demonstration. In: . (Orgs.). Community Organizing: building social capital as a development strategy. 1998. Disponível em: <http://dx.doi.org/10.4135/9781452220567.n2> Acesso em: 14 mar. 2018.

GREEN, Gary; HAINES, Anne. The role of assets in community-based development. Sage Publications $\quad$ Inc. $2008 . \quad$ Disponível em: <http://www.sagepub.com/sites/default/files/upmbinaries/39151_Chapter1.pdf>. Acesso em: 25 mar. 2018.

. Asset Building \& Community Development. Sage Publications Inc. 2012.

GUDYNAS, Eduardo. Ecología, economía y ética del desarrollo sostenible. 2004. Disponível em: < http://www.ecologiapolitica.net/gudynas/GudynasDS5.pdf>. Acesso em: 11 ago. 2018.

INE. Instituto Nacional de Estadísticas. La mujer en la agricultura chilena. Resultados de Censo Agropecuario. 2007.

IPCC. Intergubernamental Panel For Climate Change. Managing the risks of extreme events and disasters to advance climate change adaptation. Special Report. 2012. Disponível em: <https://www.ipcc.ch/pdf/special-reports/srex/SREX_Full_Report.pdf>. Acesso em 20 abr. 2018.

KEUCHEYAN, Razmig. Estado, capitalismo y naturaleza. La expansión del "mercado de las catástrofes". 2014. Disponível em: <http://nuso.org/articulo/estado-capitalismo-y-naturalezalaexpansion-del-mercado-de-las-catastrofes/>. Acesso em: 21 abr. 2018.

KNUDSEN, Brian; FLORIDA, Richard; ROUSSEAU, Denisse. Bridging and bonding: a multidimensional approach to regional social capital. In: Ontario Network on the Regional Innovation System Newsletter. 2008 Disponível em: $<$ http://www2.rotman.utoronto.ca/userfiles/prosperity/File/Bridging_and_Bonding.w.cover.pdf>. Acesso em: 22 mar. 2018.

LEFF, Enrique. La ecología política en América Latina: un campo en construcción. Brasilia: Sociedade e Estado. 2003.

LIN, Nan. A network theory of social capital. Handbook of social Capital. Oxford University Press. 2008.

LINDELEY, Anne. Cuestionando el "desplazamiento a causa de la sequía”medioambiente, política y migración en Somalia. RMF. n. 45, 2014. Disponível em: <http://www.fmreview.org/es/crisis/lindley.pdf>. Acesso em: 21 abr. 2018. 
MITTELMAN, James. Globalization and environmental resistance politics. In: PRAZNIAK, Roxann (Org.). Places and politics in an age of globalization. Rowman \& Littlefield Publishers. 1997.

NARAYAN, Deepa. Bonds and bridges: Social capital and poverty. World Bank. 1999. Disponível em: <https://info.worldbank.org/etools/docs/library/9747/narayan.pdf>. Acesso em: 23 mar. 2018.

NARAYAN, Deepa; CASSIDY, Michael. A dimensional approach to measuring social capital: development and validation of a social capital inventory. Banco Mundial - Red de gestión económica y de reducción de la pobreza. 1999.

OECD. Organizacion para la Cooperación y el Desarrollo Económico. What is social capital? In: OCDE insights: human capital. OCDE. 2007. Disponível em: <http://www.oecd.org/insights/37966934.pdf>. Acesso em: 23 mar. 2018.

O’ CONNOR, James. Causas naturales. Ensayos de marxismo ecológico. México: Siglo XXI. 2001.

OSTROM, Elinor. Governing the commons: the evolution of institutions for collective actions. Cambridge University Press. 1990.

PENGUE, Walter. La economía ecológica y el desarrollo en América Latina. Revista de la Universidad de Buenos Aires. v. 7, n. 14, 2012. Disponível em: <https://www.ungs.edu.ar/wpcontent/uploads/2012/07/6.2Econom\%C3\%ADaEcológica_Walter-Pengue.pdf>. Acesso em: 15 ago. 2018.

PEREDA, Carlos; DE PRADA, Miguel Ángel; ACTIS, Walter. Investigación acción participativa: propuesta para un ejercicio activo de la ciudadanía. In: FERRER, Juliana; PELAKAIS, Cira de; AGUIRRE, René (Orgs.). Proceso operativo de los servicios comunitarios en las universidades. Kan Sasana Printer. 2008. Disponível em: <http://www.investigaccio.org/ponencies/IAP.pdf>. Acesso em: 25 maio 2018.

PUTNAM, Robert. Making democracy work: civic traditions in modern Italy. 1993. Disponível em: <http://www.bestbooklibrary.com/signup?b=1\&ad_domain=ads.adcenter.com\&ad_path=/smart_ad/ display\&prod=176\&ref=5045567\&q=making\%20democra

cy\%20work\%20civic\%20traditions\%20in\%20modern $\% 20$ italy\&seed=3593081233\&sf=bo

oks_newdesign\&utm_source=grafit.lv\&utm_medium =referral\&placement=grafit.lv\&adser ver $=0.17 .0-\mathrm{rc} 5 \& \mathrm{~m}=$ books\&sid=747\&bt=1444344859623\&bh=1192517760 >. Acesso em: $25 \mathrm{mar}$. 2018.

PUTNAM, Robert. Bowling alone: the collapse and revival of american community. Simon and Schuster. 2000.

RAM, Ganga; PRASAD, Krishna. Bridging, linking and bonding social capital in collective action. The case of Kalahan Forest Reserve in the Phillippines. Capri Working Paper. n. 79, 2008. Disponível em: <http://ebrary.ifpri.org/cdm/ref/collection/p15738coll2/id/11647>. Acesso em: 22 maio 2018.

SALANOVA, Margarita, CABANEÉS, Eurídice. Arte versus globalización: Revisión filosófica de los recursos del arte contemporáneo frente al proceso de globalización. 2010

TELLERIA, Nelson; CONDE, Carmen. La cestería indígena en la cultura ancestral Venezolana. Investigación y Postgrado. Investigación y Postgrado. v. 19, n. 2, p. 213-228, 2004. Disponível em: <http://www.scielo.org.ve/scielo.php?script=sci_arttext\&pid=S1316-0087200400>. Acesso em: 5 maio 2018. 
34 | Carolina Fuentes Lizama

VALDIVIESO, Patricio. Municipal governance, environmental management and disaster risk reduction in Chile. Bulletin of Latin American Research. v. 36, n. 4, p. 440-458, 2016.

VALDIVIESO, Patricio; DAVIDOVICS, Gabriel. Enfoques y metodologías para la investigación empírica del capital social. Santiago: Universidad Central. 2016.

Texto recebido em 25 de junho de 2018. Aprovado em 11 de agosto de 2018. 